\title{
Vedic Systems and their Scientific Aspects to Sustain Mental Health: A Solution for Challenging Issues for Human Race in Epidemic Time
}

\author{
Rohit Rastogi ${ }^{1 *}$, Sheelu Sagar ${ }^{2}$, Neeti Tandon $^{3}$, Rajeshwari $\mathrm{T}^{4}$ and Luv \\ Dhamija $^{5}$ \\ ${ }^{1}$ Sr. Assistant Professor, Department of CSE, ABES Engineering College Ghaziabad, U.P, India \\ ${ }^{2}$ Research Scholar, Amity International Business School, Amity University, Noida, U.P, India \\ ${ }^{3}$ Research Scholar, Vikram University, Ujjain, M.P, India \\ ${ }^{4}$ Yagyopathy Researcher and Active Social Volunteer, Kolkata, W.B, India \\ ${ }^{5}$ Students, B.Tech. CSE Third Year, ABES Engineering College Ghaziabad, U.P, India
}

Research Article

Volume 5 Issue 1

Received Date: January 14, 2021

Published Date: February 16, 2021

DOI: $10.23880 /$ jonam-16000299

*Corresponding author: Rohit Rastogi, Sr. Assistant Professor, Department of CSE, ABES Engineering College Ghaziabad, U.P, India, Tel: +91-8076772048; 9818992772; Email: rohit.rastogi@abes.ac.in

\section{Abstract}

The Mental Fitness has been found as a great threat after pandemic in $21^{\text {st }}$ Century world. People are in trouble due to anxiety, stress and depression because of many reasons and it also reduces their productivity and work efficiency. The Researcher team has conducted a survey and collected the data from different parts of the world and analyzed it critically. Apart from visualizing the stress related responses, the team had found the effect of Indian Ancient Mantra and Yoga along with Pranayama through a specified protocol to identify its benefits in the mental fitness, survival in tough conditions and come out of bad experiences. The Data Analysis has been done through various related tools and scientific explanation has been established.

Keywords: Yajna; Mantra; Stress; Depression; Anxiety; Mental Fitness

\section{Introduction}

The Various Scientific Aspects and Yajna and Mantra are specified below.

\section{The Diseases and Subtle Power of Yajna}

\section{$>$ Weakness of will is Reason of Diseases}

Due to the weakness of will, nowadays everyone considers himself to be suffering from some disease. Many of us are so upset that it is as if they are suffering from some terrible disease but in reality; this is not the case. With the help of strong will, the attack of big diseases can also be avoided and your health can be protected. There is also the subtle science of Yajna. Such elements exist in this philosophy and law which increases the amount of deity in the invisible atmosphere of the microcosm. It helps in all-natural healing, peace and peace [1].

\section{$>$ Pragya Yoga}

Pragya Yoga is required by an individual not less than three rounds. Meditation should be done with chanting of selected and validated Mantra. The mixing of both chanting and meditation should be understood. One should pay attention that Savitri and Gayatri are two goddesses. One must witness the morning Sun under Gayatri. This is universal (Amritvani of Pujyavar, Shriram Sharma Acharya) [2].

\section{$>$ Worship of Sun}

After chanting, Sun Arghyadan is performed, to God Surya, you offer water to the Sun in front of the Sun. This 


\section{Journal of Natural \& Ayurvedic Medicine}

is surrender; Surrender to God, Savita, to Surya. If you are chanting in the morning, then offer water in the east direction. If you are chanting in the evening, then offer water in the west direction

$[3,4]$.

\section{$>$ Yajna and Gayatri Importance}

Due to the dirt coming out of the human body continuously, the air system which gets contaminated, its purification is done with the aroma of Yagya. If we humans create a fragrance by wearing a body and produce the same fragrance, we get rid of the crime of contaminating the public air element (Yagya: A Holistic Healing Process, Yuga Rishi Shriram Sharma Acharya) [5].

The properties of medicine through Yajna are made very subtle, one can experience with spiritual practices, some sprinkling of the power of this subtlety. One can easily view the process of pharmacology of homeopathy in Yajna. On the basis of this invention, it has happened that the power of the drug is increased many times more when it is more subtle (Yugrishi Shriram Sharma Acharya, Yagya Pita- Gayatri Mata).

The power deities generated by Yajna makes everyone strong and satisfied with that aroma and it organizes all the works from creation to creation to world welfare (Padma Purana--Yajna: A Holistic Healing Process, Yugrishi Shriram Sharma Acharya) [6].

http://ijyr.dsvv.ac.in/index.php/ijyr/article/ view/11/25. In the images below, the volunteers of Gayatri Parivar explains the scientific aspects of Yajna, Mantra and meditation etc. to common public in very easy and friendly language (Figure 1).

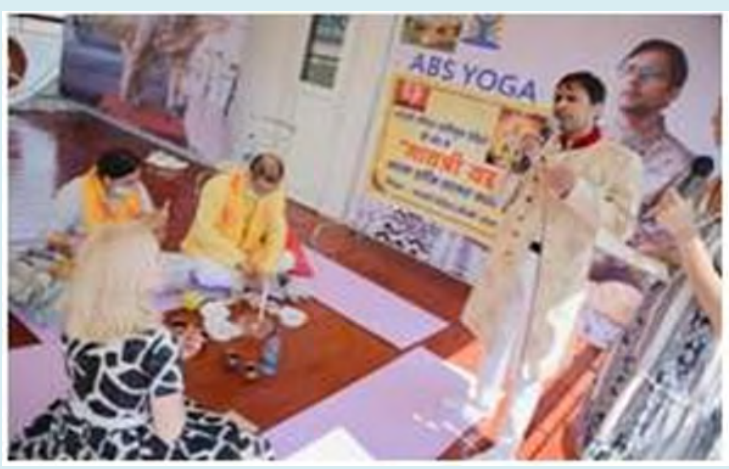

Figure 1: The Volunteers of All world Gayatri Parivar explaining the scientific aspects of Yajna and Mantra

\section{$>$ Yajna Cures Diseases and Germs}

Yajna is called as father and Gayatri and mother. Yagya: The powerful elements which are spread in the atmosphere through the Yagya, innumerable disease germs are easily destroyed by moving around in the air. Yogi performs more effective remedies than spraying of D.D.T., Finial etc., to prevent diseases (Yajna: a healing process, Yug Rishi Shriram Sharma Acharya) [7].

Also one can see below that in Indian subcontinent, people prefer the Gayatri Yajna and Rudrabhishek in dealing with Covid'19 (Figure 2). Air-conditioned medicines work as much as they can do, which can't be done by eating and drinking (Yajna: a holistic healing process, Yug Rishi Shriram Sharma Acharya). Divine powers are subtle, so to reach them subtle is needed. Sacrificial objects make it subtle. The Yajna coordinates in the three worlds, namely, the heavens, the earth and the body, and this concord results in an empire of happiness and peace (Yajna: a holistic healing process, Yugrishi Shriram Sharma Acharya) [8].

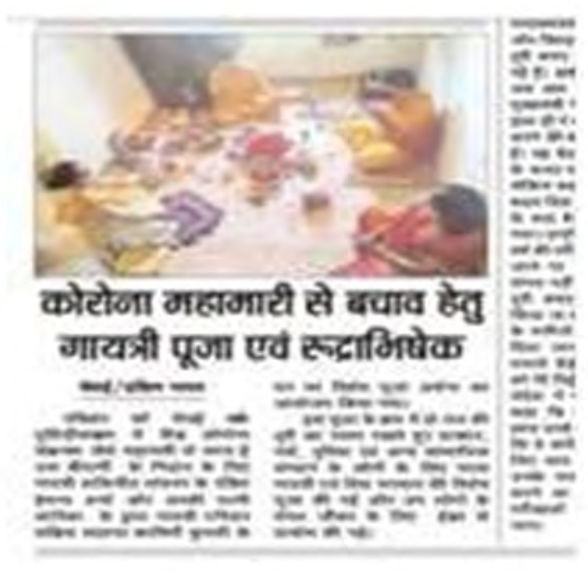

Figure 2: Gayatri Mantra and Rudrabhishek is effective in Covid'19.

Burning nutmeg, mace, large cardamom, and dry sandalwood found that their oil spreads in the air as gaseous, the fire burns them to gas. Its atoms are very subtle, measured by micro-spectator, then this atom is from $1 / 10000$ smaller ones up to $1 / 100000000 \mathrm{~cm}$. diameter were found. Dr. "Hafkin" is noted as the inventor of the Smallpox vaccine. Thus an equitable treatment process (Yug Rishi Shriram Sharma Acharya, Yagya- a holistic healing process).

In the aspects of Yagya, playing with the rules of the nature; the one doing rituals can never be a patient, it a sure opinion described in the Vedas and tuberculosis (lungs disease) is scared to even come near the house of this person. With the help of thousands of powers, giving 100 years to live, this Hawan helps the patient and protect him from the claws of death. _May god free us from all the sins and diseases', (Atharva Veda 3/11/13)(Yagya: a holistic healing process, Yug Rishi Shriram Sharma Acharya) $[9,10]$.

The Meditation and Pranayam along with Yajna and Mantra Chanting is much more useful in relief of stress, 
diabetes and many more human health threats. Latest instruments along with sensors are recording positive reflections on it (Figures 3-5).

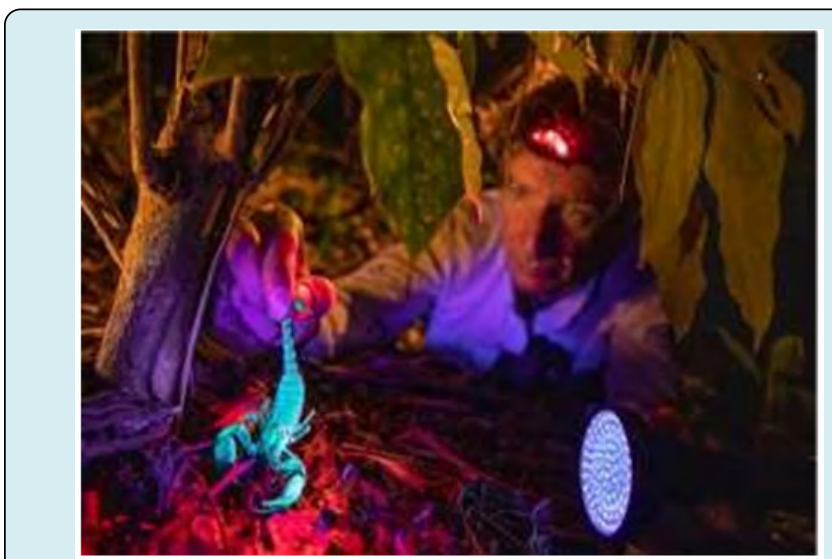

(A World of Pain", National Geographic Magazine, vol. 1, issue-1, pp. 32-57, (January 2020)), Source:https://www. nationalgeographic.com/magazine/2020/01/scientistsare-unraveling-the-mysteries- of-pain-feature/

Figure 3: Zolta $n$ Takacs, a biomedical scientist and National Geographic explorer, and Nguyen Thien Tao, a herpetologist with the Vietnam National Museum of Nature, searched for toxic creatures in Chu Yang Sin National Park in Vietnam's Central Highlands. Hoping to identify compounds that could lead to new pain medications, the two scientists captured scorpions, snakes, snails, frogs, centipedes, and spiders. (Courtesy: National Geographic Magazine, Jan. 2020).

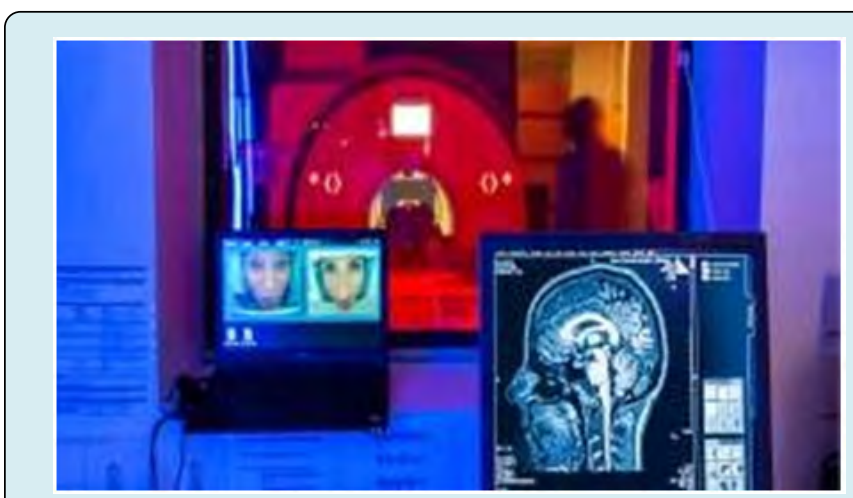

(A World of Pain", National Geographic Magazine, vol. 1, issue-1, pp. 32-57, (January 2020)),

Source:https://www.nationalgeographic.com/ magazine/2020/01/scientists-are-unraveling-themysteries- of-pain-feature/

Figure 4: The relationship a patient has with a doctor can affect how much pain the patient feels, Napadow said, "but we don't know why that is." To explore this phenomenon, Napadow simultaneously records the brain activity of an acupuncturist and a patient in separate functional magnetic resonance imaging machines.

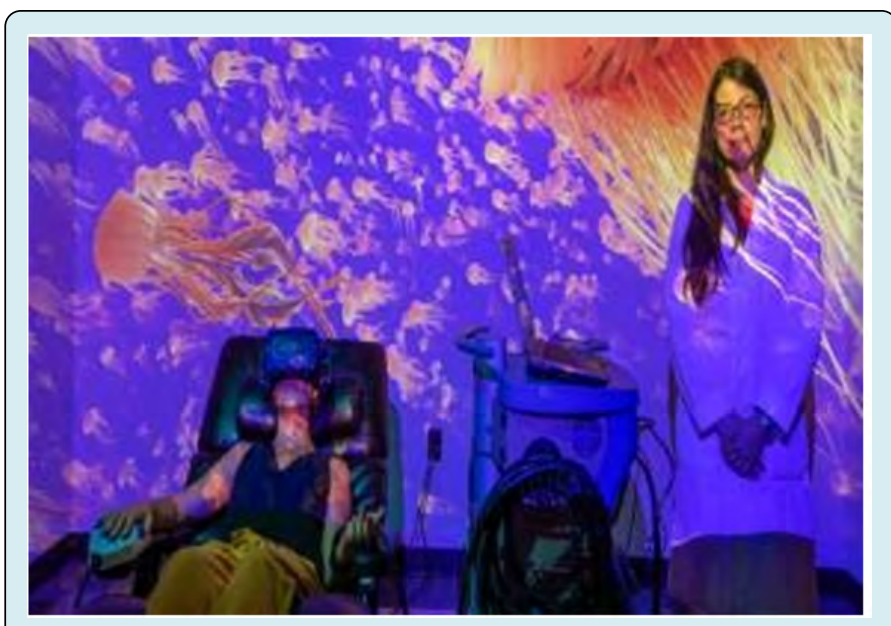

(A World of Pain", National Geographic Magazine, vol. 1, issue-1, pp. 32-57, (January 2020)), Source:https://www. nationalgeographic.com/magazine/2020/01/scientistsare-unraveling-the-mysteries- of-pain-feature/

Figure 5: Vitaly Napadow, a neuroscientist at Harvard Medical School and Massachusetts General Hospital, studies how the brain perceives pain. To do that, he uses electroencephalography to track the brain wave patterns of patients with chronic lower back pain.(Courtesy: National Geographic Magazine, Jan. 2020).

\section{Literature Survey}

\section{Yajna and Environment}

Today the whole world suffers from the odd problem of environment. All living organisms spread dirt through urine and the industrialization has become the culmination of dirt. Man is increasing his means of happiness and destroying the environment in the same proportion. Pure air and water are mainly necessary for the life on earth to remain. Today, neither pure air nor water is available due to the filth that surrounds it. As the human mind is getting dirty, the environment prevailing around it is also getting contaminated $[11,12]$.

It is easiest to purify the environment with a Yajna. Smoke picked up from the sacrificial fire is spread around by the gusts of wind and the elements of the substance in the form of microscopic particles are also spread around and they enter the body of the stripes with the breath. Also, tree plants are also affected by it. Havan gas has many useful chemical elements. It is illusion that the smoke of the incense contains carbon dioxide and any other toxic gas. Havan gas is devoid of this defect. Even if there are some toxic parts, the evaporative effect of the melt makes it beneficial by destroying it. Many chemical aromatic substances located in it cleanse the environment by removing all types of pollution. In addition to purification of air, Havan gas purifies many elements such as space, water, etc. 


\section{Journal of Natural \& Ayurvedic Medicine}

This gas, combined with air, rains the earth in the form of rain. Through it, the water source is made pure, the vegetation is satisfied, the fertile power of the soil increases. The earth of the Yajna absorbs the smell of the smoke of the Yajna. This increases the agricultural yield of the Earth manifold and also becomes nutritious $[13,14]$.

Statistics show that the fertility of the soil is greatly increased by the gas produced by the Yajna. Today, anomalies are spreading around the environment. Ozone layers of the atmosphere are bursting due to pollution. In such a situation, the utility of Yajna is more than ever Just as iron cuts iron, a thorn comes out of a fork; similarly a thorn of misconceptions is coming out by spreading good ideas in the idea revolution campaign. Similarly, smoke can also be purified with smoke. Toxic gases are circulated in the atmosphere. They can be purified only by sacrificial smoke and there is no way. Group activities to perform these are much beneficial $[15,16]$ (Figure 6).

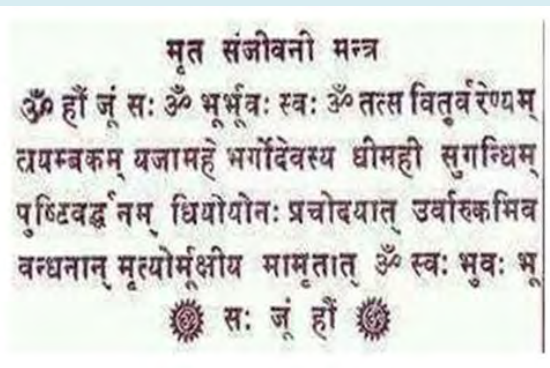

Figure 6: Mrit Sanjeevani Mantra to cure inevitable Diseases.

\section{Experimental Setup and Methodology}

The Experiments to support the science of Yajna and Mantra were conducted through a well defined protocol of 45 minutes where the Mantra Chanting, Pranayama, Yoga and Fitness activities were carried out. The laughing therapy and some alternative therapies like acupressure, Pranik Healing were also used in different time intervals.

\section{Protocol Followed}

The readings were being recorded by research team members quantitatively under different parameters and analyzed through various data analytics tool like Python, Tableau, SPSS, and excel. The subjects were asked to fill a small consent form to give their will to participate in the aforesaid experiments and were assured that their identity and data will not be made commercial and misused.

The whole experiment was conducted through online platforms like Google meet, Zoom sessions, MS Team etc. as per convenience of the subjects during the lockdown period and their scores were recorded. The activities were conducted in India of duration of pandemic Sars-Cov'19 peak of $1^{\text {st }}$ April 2020 to $31^{\text {st }}$ Aug. 2020 in the period of 150 days around where a strict lockdown was imposed in India to save the mass from third stage infection of spread of Sars-Cov'19.

The Subjects were asked to follow a strict protocol of 45 min. around which included some Asans ( sitting, standing, lying front and back postures), some set of Mantra Chanting, Breathing Exercises( Pranayama), Alternate Therapies like Laughing Technique and Shantipath with certain breathing Exercises were followed. Subjects'data was recorded pre and post of the experiment and accordingly comparative study was conducted to check the efficacy of the methodology adopted $[17,19]$.

If this Mantra is chanted 12 times a day then one'll be protected from all natural disasters. After a cyclone in Shantikunj-Haridwar, an old man told us about this and wrote it down with his own hands (Figures 7,8).

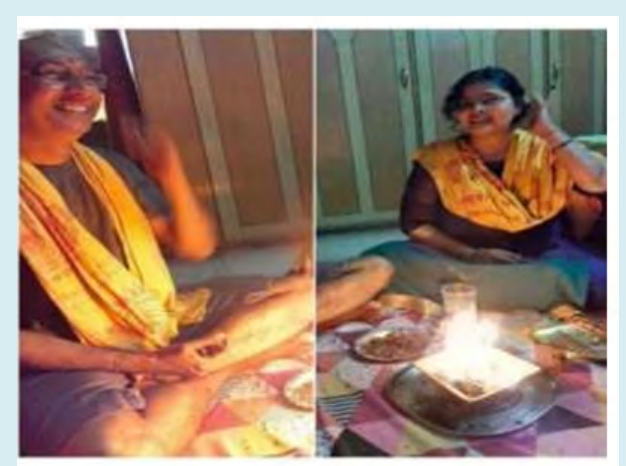

Figure 6: Yajna Rituals Performed by Volunteers of All world Gayatri parivaar.

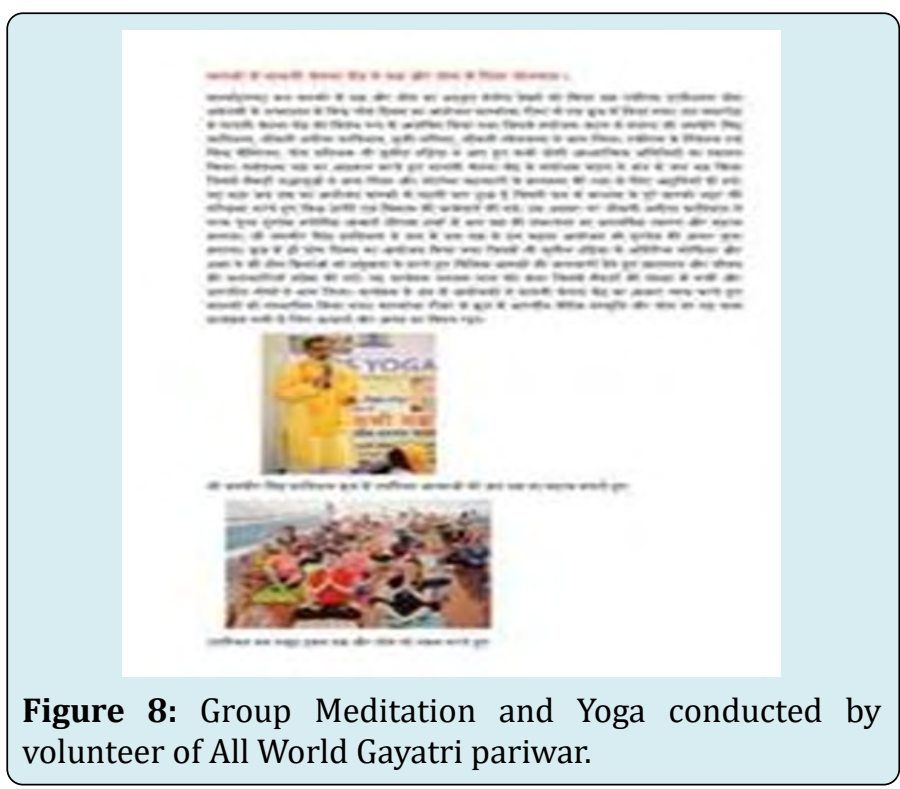




\section{Yajna Items/ Components for different Properties}

For Different Satogun, Rajogun and tamogun, different ite,s and components are used in Yajna. RajoGun-MalaChandan, Asan-Soot, Flower-Yelllow, Vessel-Kansa, ClothResham, Direction-North, Ghee in Deepak- of bufello, Tilak- Roli, Samidha in Yajna- Mango, Dhak, Shessham, Havan Samagri components: Badi Ilayachi, Keshar, Chaar Chabila, Punarnava, Jeevanti, Kachoor, Talis Patra, Rasna, Nagarmotha, Unnav, Talamkhana, Mochras, Saunf, Chitrak, Daalchini, Padmakh, Chhuara, Kishmish, rice, Khand [20,22].

\section{No use of Salt in Yajna}

In the language of chemistry, salt is called sodium chloride. Chlorine is a poisonous gas, whose slight gust is enough to make a human unconscious for 24 hours, a small amount can also transport it to other folk, hence salty or salted items are not used in the Yajna (Book of the most revered Gurudev Pt. Sriram Sharma Acharya ji, are you not eating poison?). Other reasons for this is that the salt melts when burnt and the water element increases, making it difficult for the fire to ignite. It becomes an impediment to burning. In the Yajna, objects of oily nature are made so that there is convenience in burning.

The chemical formula of salt is NACL NACL and when the temperature in the fire is above 250 degrees centigrade, sodium breaks down to form oxides and chlorine breaks down and releases chlorine gas into the atmosphere, which is a toxic gas. If salt will be put into the yagna or sacrificed with salty things, there will be burning of poisonous gas as well as the skin. That is why a yajna is prohibited with a salty item or salt. Likewise, it is forbidden to perform yajna on neem wood because when neem wood burns, it also releases chlorine in small amounts in small amounts. That is why sacrificial fire with neem wood also has a bad effect on the skin.

\section{Results and Discussions}

\section{Case Study of a Diabetic Patient and Aayurved Treatment}

A lady who was suffering from Diabetes from the past two years and had been bound to take insulin injections twice a day is now finally free from all the restrictions. Yes, she is free from the abnegation of sweets too. The doctors have also advised the lady to stop taking blood-sugar control medicines and insulin. The distinguishing feature about these medicines is that, since they're home-made they don't have any side-effects. A kidney specialist from Mumbai, Dr. Tony Almeda, has used these medicines ambient and has found them to be wonderful. Hence, it is suggested to everyone that all use this medicine and get lots of benefits from them. The requirements are-Wheat (100 grams), Cellulose of a tree (100 grams), Barley (100 grams), Margraila (100 grams).

\section{Recipe to make it}

Mix these items with 4 cups of water, Boil itfor 10 minutes, and let it cool itself. After it cools down, filter it and keep it safely in a bottle. How to use it-A small cup of it should be taken daily for a week(empty stomach).From the next week, it should be taken in alternate days(empty stomach).After 2 weeks, an astonishing change will be noticed in oneself. After using this, one can eat a proper diet $[23,25]$.

\section{Results on Stress Analysis}

After School, the largest teen-focused social network, polled current high school students from around the U.S. over several days on the topic of stress and mental health and their research is present in data. World site on stress plays a major role in the overall mental health of a teen. As a follow up to their Social Media Safety in Schools 2018, Conference on teen suicide prevention and mental health, the organization asked their users how much of a role stress plays in their lives, and how beneficial to manage it. They used this information to raise awareness about the state of teen mental health. A total of 35,878 teens participated in the poll (Figures 9-13).

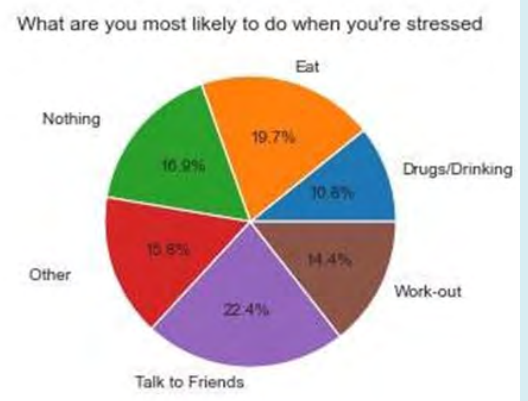

Figure 9: Pi Chart on Survey of doing activities while being in Stress.

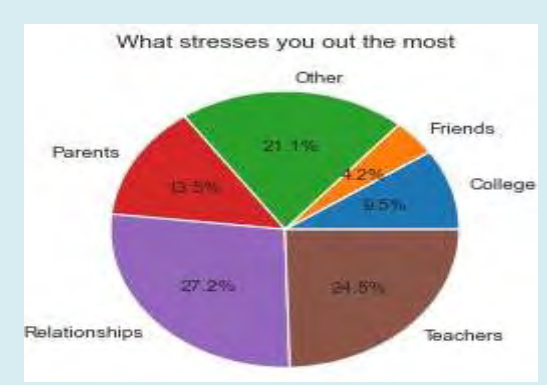

Figure 10: Pi chart to depict the reason of Stress. 


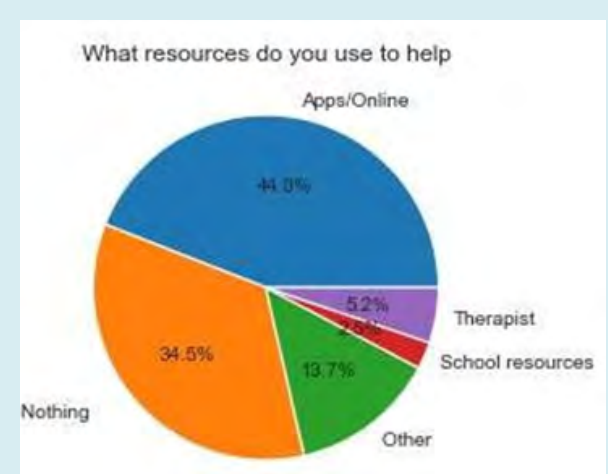

Figure 11: Pi chart to know the resources to help in stress.
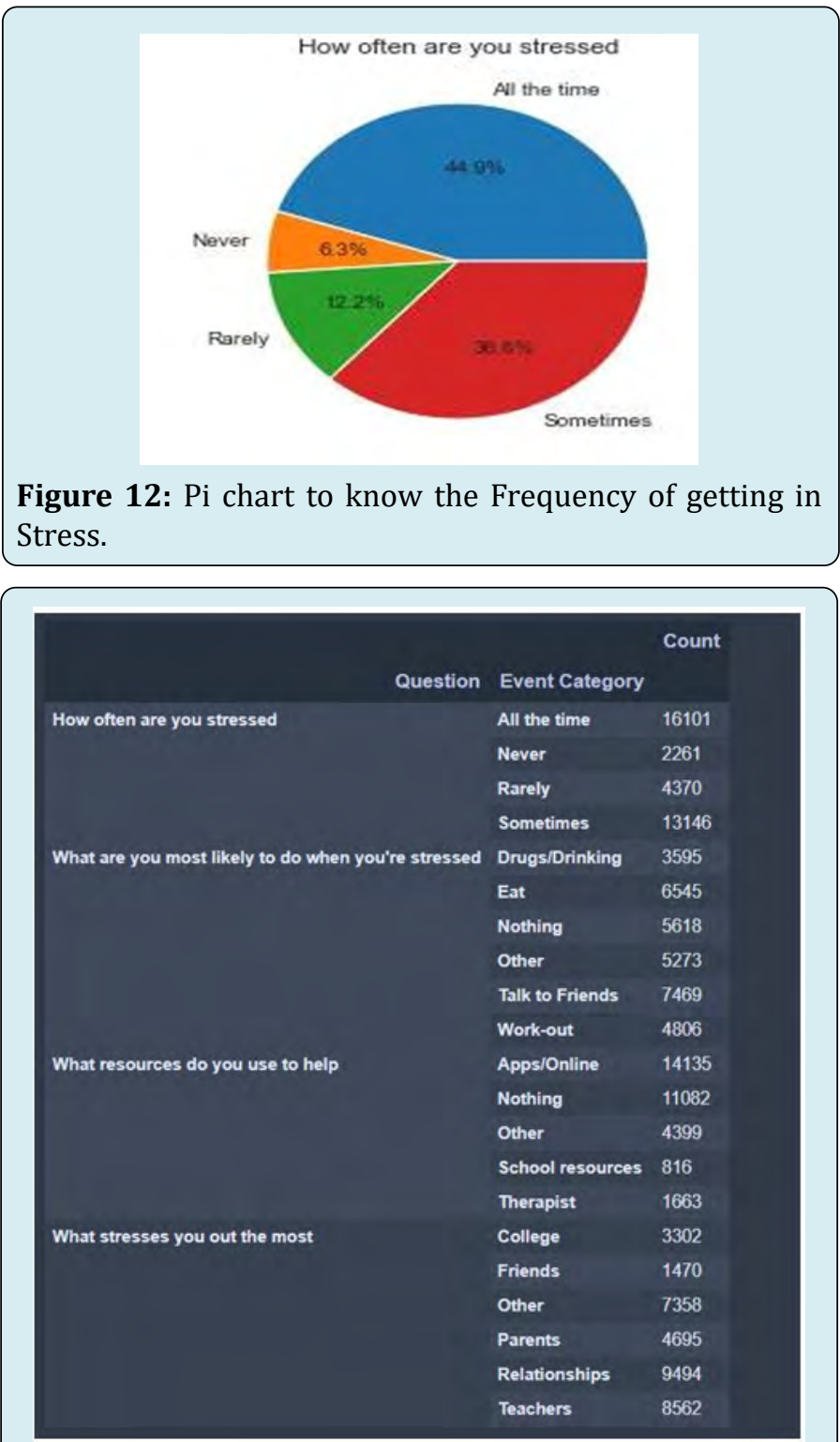

Figure 13: The responses of different participants in Stress related questionnaire

\section{Recommendations}

Prediction, Pledge and Assurance by Acharya Ji; is that it is highly recommended for the manuscript present here that we might not live perfect, but we will live in a subtle body, that way we will be able to walk 20 thousand miles in one minute. Our subtle body is unlimited. Our subtle body can do more work that our body is made of clay and mud. Don't worry if the Gurudev is dead and what you will do now, Son, don't worry we will reach to you ourselves, to supervise you, to protect you, keep you healthy and give you the path of progress. We will be your umbrella in this odd situation (Yugrishi Shriram Sharma Acharya ji).

We give a portion of our tenacity to others in grief, to wipe their tears. So why sir! Will you be in loss? What do you talk? You check the letters that we have written and sent. We have overcome the suffering of people with honesty. We get a thousand letters, out of which nine hundred are only with sorrow and troubles. We remove their problems and wipe their tears from their eyes (Yug Rishi Shriram Sharma Acharya ji). Nobody in the world is as great as Maa Gayatri. Gayatri Maa has in carnated, she will be the Yug Shakti. The time has come, the era will change, it will recognize as the Kaal Shakti (Yug Rishi Shriram Sharma Acharya ji) [26-30].

\section{Novelity}

Indian Culture and Gayatri related scientific analysis is the main novelty of the content. Agnihotra: It is not only a recognized religious practice of Indian culture, but also includes many scientific facts behind it. On this basis, where the Indian Science serves the purpose of self-realization, it's physical response also fulfills its physical and mental purposes. Lost health doesn't return the repository of biographical power is replenished, the attack of disease germs, viruses decreases and is canceled (Yajna: a holistic healing process, Yugrishi Shriram Sharma Acharya) [31,32].

\section{Future Research Directions}

Gayatri is also worshiped as Sandhya Vandan and daily as Agnihotra. The above five sacrifices should be carried out in our entire family. There should be an atmosphere of Gayatri worship in the every family and sacrifice as global. If Agnihotra continues, it will be understood that the Puja Vidhan of Gayatri Mata and Yagya Daddy is going on well. Research Has to be done to establish the benefit of each ritual and activity performed here. (Gayatri MahaVigyan-Yugrishi Shriram Sharma Acharya) [33-35].

\section{Conclusion}

The need of the change is the demand of era. Nowadays, in which life has reached an unethical level, it is no longer 


\section{Journal of Natural \& Ayurvedic Medicine}

possible for small reformers to work with small reforms, now it has become necessary for it to have a lot of upheaval, turmoil. The element -Mahakal - has been repatriated by God to fulfill this purpose. They are still going to do the same. (Mahakal and Yug conversion process, by Yugrishi Shriram Sharma Acharya ji, Shantikunj Haridwar). Our reverse intellect is making heaven hell. Our brain is overcome by this odd situation. (Gayatri MahaVigyan to straighten the Time, Yugarishi Shri Ram Sharma Acharya).

\section{Acknowledgement}

The Team of authors would love to pay our deep sense of gratitude to the ABES Engineering College, Ghaziabad \& Amity International Business School, Amity University, and Noida for arranging us all the facilities, the direct-indirect supporters for their timely help and valuable suggestions and the almighty for blessing us throughout. We would also like to extend the vote of thanks to IIT Delhi, IIT Roorkee, Dev Sanskriti Vishwavidyalaya, Haridwar, Patanjali Foundation and Ayurveda Institute, Dehradun for their support and guidance in accomplishing our research paper.

\section{References}

1. Cameron AJ, Zimmet PZ, Dunstan DW, Dalton M, Shaw JE, et al. (2003) Overweight and obesity in australia: the 1999-2000 australian diabetes, obesity and lifestyle study (ausdiab). The Medical Journal of Australia 178(9): 427-432.

2. Chaturvedi DK, Arya M (2013b) Correlation between human performance and consciousness, ieee-international conference on human computer interaction, Saveetha School of engineering, Saveetha University, Thandalam, Chennai, IN, India.

3. Chaturvedi DK, Arya M (2013a) A Study of Correlation between consciousness level and performance of worker. Industrial Engineering Journal 6(8): 40-43.

4. Chaturvedi DK, Satsangi R (2013) the correlation between student performance and consciousness level, international conference on advanced computing and communication technologies (ICACCT ${ }^{\mathrm{TM}}$-2013), Asia Pacific Institute of Information Technology SD India, Panipat (Haryana), Souvenir, pp: 200-203.

5. Gunavathi C, Premalatha K (2014) A Comparative analysis of swarm intelligence techniques for feature selection in cancer classification. The Scientific World Journal 14: 12.

6. Jain G (2017) BLOG- Hawan for cleansing the environment.
7. Kim KJ, Tagkopoulos L (2019) Application of machine learning rheumatic disease research. Korean J Intern Med 34: 2.

8. Lahoty P, Rana M (2013) Agnihotra organic farming, Popular Kheti 1(4): 49-54.

9. Mahajan P (2016) Application of pattern recognition algorithm in health and medicine: a review. International Journal of Engineering and Computer Science 5(5): 16580-16583.

10. Marjani M, Nasaruddin F, Gani A, Karim A, Hashem IAT, et al. (2017) Big IoT data analytics: architecture, opportunities, and open research challenges. IEEE Access 5: 5247-5261.

11. Mistry R, Tanwar S, Tyagi S, Kumar N (2020) Blockchain for $5 \mathrm{G}$-enabled iot for industrial automation: a systematic review, solutions, and challenges, mechanical systems and signal Processing 135(1): 1-19.

12. Shenwai MR, Tare KN (2017) Integrated approach towards Holistic Health: Current Trends and Future Scope. Int J of Cur Rec Rev 9(7): 11-14.

13. Rastogi R (2012) Current Approaches for Researches in Naturopathy: How far is its Evidence Base? J of Homeopathy \& Ayurvedic Medicine 1(2): 1-107.

14. Rastogi R., Chaturvedi DK, Sharma S, Bansal A, Agrawal A (2018a) Audio visual EMG \& GSR biofeedback analysis for effect of spiritual techniques on human behaviour and psychic challenges. In Proceedings of the 12th INDIACom, pp: 252-258.

15. Rastogi R, Chaturvedi DK, Satya S, Arora N, Sirohi H, et al. (2018b) Which one is Best: Electromyography Biofeedback Efficacy Analysis on Audio, Visual and Audio-Visual Modes for Chronic TTH on Different Characteristics, in the proceedings of International Conference on Computational Intelligence \& IoT (ICCIIoT) 2018, at national institute of technology Agartala, Tripura, India, ELSEVIER- SSRN Digital Library.

16. Rastogi R, Chaturvedi DK, Satya S, Arora N, Saini $\mathrm{H}$, et al. (2018c) Comparative efficacy analysis of electromyography and galvanic skin resistance biofeedback on audio mode for chronic tth on various indicators, in the proceedings of International Conference on Computational Intelligence \& IoT (ICCIIoT) 2018, at National Institute of Technology Agartala, Tripura, India, ELSEVIER-SSRN Digital Library (ISSN 1556-5068).

17. Rastogi R, Saxena M, Sharma SK, Muralidharan S, Beriwal VK, et al. (2019a) Evaluation of efficacy of yagya therapy 
on t2- diabetes mellitus patients, in the proceedings of The $2^{\text {nd }}$ (Edn.), of International conference on industry interactive innovations in science, engineering and technology (I3SET2K19) organized by JIS College of Engineering, Kalyani, West Bengal.

18. Rastogi R, Saxena M, Gupta US, Sharma S, Chaturvedi DK, et al. (2019b). Yajna and Mantra Therapy Applications on Diabetic Subjects: Computational Intelligence Based Experimental Approach, in the proceedings of The $2^{\text {nd }}$ (Edn.), of International Conference on Industry Interactive Innovations in Science, Engineering and Technology (I3SET2K19) organized by JIS College of Engineering, Kalyani, West Bengal.

19. Rastogi R, Saxena M, Sharma SK, Murlidharan S, Berival VK, et al. (2019c) Statistical analysis on efficacy of yagya therapy for Type- 2 diabetic mellitus patients through various parameters, In: Das A, et al. (Eds.), on Computational Intelligence in Pattern Recognition (CIPR), Kalyani, West Bengal. Advs in Intelligent Syst, Computing, Vol. 1120, Computational Intelligence in Pattern, Recognition.

20. Rastogi R, Chaturvedi DK, Verma H, Mishra Y, Gupta M (2020a) Identifying Better? Analytical trends to check subjects'medications using biofeedback therapies, igl global. International Journal of Applied Research on Public Health Management.

21. Rastogi R, Gupta M, Chaturvedi DK (2020b) Efficacy of study for correlation of th vs age and gender factors using emg biofeedback technique. International Journal of Applied Research on Public Health Management 5(1): 49-66.

22. Rastogi R, Chaturvedi DK, Satya S, Arora N, Gupta, M, et al. (2020c) An optimized biofeedback EMG and GSR biofeedback therapy for chronic TTH on SF-36 scores of different mmbd modes on various medical symptoms,in studies comp. intelligence, Vol. 841: Hybrid Machine Intelligence for Medical Image Analysis.

23. Saxena M, Sengupta B, Pandya P (2008) Controlling the microflora in outdoor environment: effect of yagya.
Indian Journal of Air Pollution Control 8(2): 30- 36.

24. Saxena M, Kumar B, Matharu S (2018) Impact of Yagya on particulate matters, interdisciplinary. Journal of Yagya Research 1(1): 01-08.

25. Saxena M, Sharma SK, Muralidharan S, Beriwal V, Rastogi R, et al. (2020) Statistical analysis of efficacy of yagya therapy on Type- 2 diabetic mellitus patients on various parameters, In Proceedings of $2^{\text {nd }}$ International Conference on Computational Intelligence In Pattern Recognition (CIPR-2020), Institute of engineering and management, kolkata, West Bengal, India.

26. Sharma SR (2015a) Shabd Brahma-Naad Brahm, BrahmVarchas, Shantikunj, pp: 98.

27. Sharma SR (2013) Shabd Brahma-Naad Brahm, BrahmVarchas, Shantikunj, pp: 55.

28. Sharma SR (2015b) Gayatri MahaVigyan, BrahmVarchas, Shantikunj, pp: 235.

29. Sharma SR (2015c) Shabd Brahma-Naad Brahm, BrahmVarchas, Shantikunj, pp: 34

30. Sharma SR (2015d) Shabd Brahma-Naad Brahm, BrahmVarchas, Shantikunj, pp: 61

31. Shrivastava V, Batham L, Mishra A (2019) Yagyopathy (Yagya Therapy) for various diseases-an overview. Ayurveda evam Samagra Swasthya Shodhamala 1(1): $1-11$

32. Srikanth (2019) 15 Benefits of machine learning in health care.

33. Srivedmata Gayatri Trust, Gayatri Parivar, UK, (2011) Yagya's effect on the environment.

34. Strate School of Design, Paris and Singapore (2016) IoT applications in healthcare, supporting Robust health and medical practices.

35. Verma S, Mishra A, Shrivastava V (2018) Yagya therapy in vedic and ayurvedic literature: A preliminary exploration.

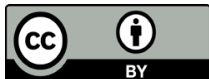

